\title{
Coagulopatias associadas à infecção por COVID-19: uma revisão de literatura
}

\author{
Coagulopathies associated with the COVID-19 infection: a literature review
}

Coagulopatías asociadas a una infección por la COVID-19: una revisión de literatura

Amanda Rocha Fernandes da Silva ${ }^{1 *}$, Beatriz Ribas de Oliveira ${ }^{2}$, Beatriz Souza Bastos ${ }^{3}$, Daniele da Rosa Fraga ${ }^{4}$, Karoline de Oliveira Lins Souto ${ }^{5}$, Maria Eduarda Fernandes Nunes ${ }^{2}$, Pedro Cesar Souza $^{6}$, Roberto Oliveira Miaki ${ }^{7}$, Yara Fernandes Ribeiro de Castro ${ }^{8}$, Bruno Augusto Lopes ${ }^{9}$.

\section{RESUMO}

Objetivo: Revisar e analisar a fisiopatologia, o diagnóstico e o manejo do paciente que apresenta distúrbios da coagulação pela COVID 19. Revisão bibliográfica: A COVID-19 provoca no organismo humano uma resposta inflamatória profunda, que tem como consequência diversos mecanismos pró-coagulantes, podendo ser considerada uma endoteliopatia que repercute em aumento da ativação plaquetária e hipercoagulabilidade, levando às manifestações clínicas pró-trombóticas associadas à infecção. A coagulopatia associada a COVID-19 apresenta nos achados típicos um aumento da concentração de dímero$\mathrm{D}$, uma diminuição modesta na contagem de plaquetas e um prolongamento do tempo de protrombina. Em pacientes de gravidade leve ou moderada é recomendado a hidratação adequada, movimentação e a profilaxia pós alta. Essa profilaxia consiste no acompanhamento do risco trombótico quando se fizer necessário. Dessa maneira, deve ser individualizada por meio da análise da idade do paciente, existência de condição de mobilidade reduzida, TEV anterior, IMC $>30$ e câncer ativo ou outras comorbidades pró trombóticas. Considerações finais: $O$ novo coronavírus provoca distúrbios respiratórios além de diversas manifestações sistêmicas como as coagulopatias. Dessa maneira, com adequado rastreio e medidas profiláticas, é possível diminuir as chances de complicações graves e melhorar o cuidado aos pacientes.

Palavras-chave: Embolia, Trombose, COVID-19, Transtornos da coagulação sanguínea.

\begin{abstract}
Objective: To review and analyze the pathophysiology, diagnosis and management of patients with coagulation disorders due to COVID 19. Bibliographic review: COVID-19 causes a profound inflammatory response in the human body, which results in several procoagulant mechanisms, which may be considered an endotheliopathy that results in increased platelet activation and hypercoagulability, leading to prothrombotic clinical manifestations associated with the infection. Typical findings in COVID-19-associated coagulopathy show an increase in the concentration of D-dimer, a modest decrease in platelet count, and a prolongation of the prothrombin time. In patients with mild or moderate severity, adequate hydration, movement and postdischarge prophylaxis are recommended. This prophylaxis consists of monitoring the thrombotic risk when necessary. Thus, it should be individualized by analyzing the patient's age, existence of reduced mobility condition, previous VTE, BMI $>30$ and active cancer or other prothrombotic comorbidities. Fina: The new coronavirus causes respiratory disorders in addition to several systemic manifestations such as coagulopathies. Thus, with adequate screening and prophylactic measures, it is possible to reduce the chances of serious complications and improve patient care.
\end{abstract}

Keywords: Embolism, Thrombosis, COVID-19, Blood coagulation disorder.

\footnotetext{
${ }^{1}$ Escola de Medicina Souza Marques (EMSM), Rio de Janeiro - RJ. *E-mail: amandarfs16@gmail.com

${ }^{2}$ Faculdade de Medicina de Barbacena (FAME), Barbacena - MG.

3 Universidade Salvador (UNIFACS), Salvador - BA.

4 Universidade do Grande Rio Professor José de Souza Herdy (UNIGRANRIO), Rio de Janeiro - RJ.

${ }^{5}$ Universidade de São Paulo (USP), São Paulo - SP.

6 Universidade Cidade de São Paulo (UNICID), São Paulo - SP.

7 Pontifícia Universidade Católica do Paraná (PUCPR), Curitiba - PR.

8 Universidade de Vassouras (UV), Vassouras - RJ.

${ }^{9}$ Hospital Municipal Miguel Couto, Rio de Janeiro - RJ.
} 


\section{RESUMEN}

Objetivo: Revisar y analizar la fisiopatología, diagnóstico y manejo de pacientes con alteraciones de la coagulación por COVID 19. Revisión bibliográfica: COVID-19 causa una profunda respuesta inflamatoria en el cuerpo humano, que da como resultado varios mecanismos procoagulantes, y puede considerarse una endoteliopatía que da como resultado una mayor activación plaquetaria e hipercoagulabilidad, lo que lleva a manifestaciones clínicas protrombóticas asociadas con la infección. Los hallazgos típicos en la coagulopatía asociada a COVID-19 muestran un aumento en la concentración de dímero D, una disminución moderada en el recuento de plaquetas y una prolongación del tiempo de protrombina. En pacientes con gravedad leve o moderada, se recomienda una adecuada hidratación, movimiento y profilaxis post alta. Esta profilaxis consiste en monitorizar el riesgo trombótico cuando sea necesario. Así, se debe individualizar analizando la edad del paciente, existencia de condición de movilidad reducida, TEV previo, IMC> 30 y cáncer activo u otras comorbilidades protrombóticas. Consideraciones finales: El nuevo coronavirus provoca trastornos respiratorios además de varias manifestaciones sistémicas como las coagulopatías. Por lo tanto, con medidas de detección y profilácticas adecuadas, es posible reducir las posibilidades de complicaciones graves y mejorar la atención al paciente.

Palabras clave: Embolia, Trombosis, COVID-19, Trastorno de la coagulación sanguínea.

\section{INTRODUÇÃO}

O novo coronavírus ou SARS-COV-2, popularmente conhecido como COVID-19, foi identificado como causador da Síndrome Respiratória Aguda Grave primariamente na China em dezembro de 2019 e anunciado como pandemia em março de 2020 pela Organização Mundial da Saúde (OMS). Até 9 de junho de 2021, foram confirmados 182.101.209 casos no mundo e 3.950.876 mortes (WHO, 2021a). No Brasil, nesse período, foram registrados 18.557 .141 infectados e 518.066 óbitos, o que justifica a grande preocupação mundial com a doença, dada sua alta morbimortalidade (WHO, 2021b).

Essa infecção contagiosa pode apresentar-se de maneira sintomática ou assintomática. Os sintomas, variáveis entre os pacientes, incluem febre, tosse seca e cansaço. Ademais, mialgia, congestão nasal, cefaleia, faringite, diarreia, disgeusia, anosmia, dispneia grave, angina, paralisia, afasia, erupção cutânea ou descoloração dos dedos das mãos ou dos pés são também manifestações clínicas comumente encontradas (HUANG C, et al., 2020). Vale ressaltar que a necessidade de admissão hospitalar foi identificada em $20 \%$ dos casos e estima-se um risco de fatalidade de $14 \%$ para pacientes de 75 anos ou mais (LEENTJENS J, et al., 2021).

Embora a COVID 19 comprometa principalmente os pulmões, órgãos como o coração, o fígado, os rins e o cérebro também podem ser acometidos (HUANG C, et al., 2020). Distúrbios de coagulação também estão frequentemente sendo observados em casos de COVID-19, principalmente em quadros mais críticos (WU C, et al., 2020). Em pesquisa realizada com pacientes gravemente acometidos pelo novo Coronavírus, foram detectados a presença de coágulos (arteriais ou venosos) nos acessos centrais em $47 \%$ dos casos, assim como trombose venosa profunda e/ou embolia pulmonar em $23 \%$ da amostra (STEFELY JÁ, et al., 2020). Ademais, uma recente meta-análise estimou uma prevalência de 14,1\% de tromboembolismo em pacientes com COVID-19 (LEENTJENS J, et al., 2021).

O SARS-COV-2 é capaz de provocar uma condição inflamatória aguda que cursa com disfunção endotelial, ativação plaquetária e hipercoagulabilidade. Esse quadro se assemelha ao da coagulação intravascular disseminada (CID) e ao de coagulopatia induzida por sepse (SIC) (IBA T, et al., 2020a). Em razão do quadro de aumento excessivo de produção de coágulos induzido pela COVID-19, há associação com complicações como coágulos arteriais e venosos, sangramentos, trombose pulmonar com microangiopatia e embolia pulmonar (STEFELY JÁ, et al., 2020; GÓMEZ-MESA JE, et al., 2021).

Diante do atual cenário de pandemia com alta morbidade e mortalidade, é essencial investigar o desenvolvimento das complicações vasculares na COVID-19, as quais podem aumentar consideravelmente o risco de morte dos enfermos e os custos para o sistema de saúde. O objetivo do estudo é revisar e analisar a fisiopatologia, o diagnóstico e o manejo do paciente que apresenta distúrbios de coagulação desencadeados pela COVID-19. 


\section{REVISÃO BIBLIOGRÁFICA}

\section{Fisiopatologia e imunogênese}

O Sars-CoV-2 é um microrganismo simples, formado basicamente por uma cápsula proteica constituída por material genético de DNA e/ou RNA (PINTO TS e DE OLIVIEIRA JB, 2021). Mutações no genoma viral do SARS-CoV-2 o tornam altamente patogênico e podem ocasionar comprometimentos em múltiplos sistemas orgânicos, além de dificultar o desenvolvimento de fármacos eficazes e vacinas (NAQVI AAT, et al., 2020). Apesar da infecção pelo Coronavírus causar principalmente injúrias pulmonares e no sistema respiratório, disfunções da coagulação também costumam ser comumente observadas nos infectados (WU C, et al., 2020).

O SARS-CoV-2 possui uma proteína $S$, que é essencial na determinação da interação hospedeiropatógeno, mediando a ligação ao receptor e a fusão de membrana para liberar RNA viral no citoplasma para a sua replicação. Durante a interação com o organismo humano, a proteína $S$ se liga principalmente ao receptor ECA2, que é expresso na superfície celular de diferentes órgãos, como coração, endotélio, fígado, rim, testículo, pulmão e outros tecidos. Dentre eles, destacam-se as células epiteliais alveolares tipo II, que abrangem $83 \%$ das células apresentadoras da ECA2 (SINGH SP, et al., 2021).

Além disso, sabe-se que na infecção pelo Coronavírus há um aumento da produção de citocinas próinflamatórias e quimiocinas, a exemplo do fator de necrose tumoral alfa (TNF- $\alpha$ ), IL-1 $\beta$ e a proteína quimioatraente de monócitos-1. Tais moléculas são responsáveis pelo recrutamento de células imunes do hospedeiro para os tecidos infectados, a fim de propiciar uma defesa que acaba causando danos ao hospedeiro. Essa ativação imunológica impulsiona a expressão do fator tecidual nas células endoteliais e nos monócitos presentes no sangue, dando início à cascata de coagulação (IBA T, et al., 2020b).

Em cerca de $20 \%$ dos pacientes esta resposta imunológica será insuficiente, devido a alterações nas respostas imunomediadas e/ou alta carga viral. A partir da evolução do quadro, tem-se agravamento dos sintomas e um extremo aumento da replicação viral, provocando apoptose de pneumócitos e células endoteliais, resultando no aumento do quadro inflamatório. Conforme há o progresso da infecção e ocorre a contínua destruição de pneumócitos, microangiopatia pulmonar e microtrombos agravando os sintomas, surge a necessidade de suplementação de oxigênio para o paciente. Em aproximadamente $5 \%$ dos casos sintomáticos o processo inflamatório irá deflagrar uma tempestade de citocinas, intensificando o quadro próinflamatório e pró-coagulatório. Essa resulta em uma endotelite sistêmica, disfunção de órgãos e celular, extravasamento capilar e ativação exacerbada da cascata de coagulação (LEENTJENS J, et al., 2021).

A COVID-19 provoca no organismo humano uma resposta inflamatória profunda, que tem como consequência diversos mecanismos pró-coagulantes. Dessa forma, a coagulopatia associada ao vírus pode ser considerada uma endoteliopatia que repercute em aumento da ativação plaquetária e hipercoagulabilidade, levando às manifestações clínicas pró-trombóticas associadas à infecção (TIBURI RGB, et al., 2021).

Além disso, por afetar diretamente as células endoteliais, que expressam ECA-2, uma enzima que faz parte do mecanismo fisiológico renina-angiotensina-aldosterona, a infecção pela COVID-19 está intimamente relacionada com a cascata de coagulação. Ou seja, quando o mecanismo renina-angiotensina-aldosterona sofre alteração, leva o sistema cardiovascular aos distúrbios de coagulação. Estudos avaliando alterações clínicas e laboratoriais vêm demonstrando presença de coagulopatia em até $50 \%$ daqueles pacientes que apresentam manifestações graves (MIESBACH W e MAKRIS M, 2020).

\section{Coagulopatia na COVID-19}

A coagulopatia associada à COVID-19 apresenta nos achados típicos um aumento da concentração de dímero-D, uma diminuição modesta na contagem de plaquetas e um prolongamento do tempo de protrombina (LEVI M, et al., 2020). A literatura demonstra uma forte associação entre a elevação dos números de dímero$\mathrm{D}$ e a ocorrência de trombose ao indicar que durante a progressiva resposta trombo-inflamatória ao Sars-cov2 ocorre uma proeminente elevação deste marcador (WANG J, et al., 2020; TIBURI RGB, et al., 2021). Ademais, os leucócitos ativados podem danificar o endotélio capilar interrompendo o estado tromboprotetor 
das células endoteliais, resultando na liberação de ativadores de plasminogênio, o que pode explicar as altas concentrações do Dímero-D como produto de degradação da fibrina na tentativa do organismo de desfazer os coágulos (CARVALHO ACS, et al., 2020; LEVI M, et al., 2020).

O Dímero-D ajuda a acompanhar a gravidade da doença e da inflamação, podendo, assim, auxiliar na avaliação do prognóstico do paciente. Da mesma forma, o tempo de protrombina (TP) alargado também é um importante marcador de gravidade (CONNORS JM e LEVY JH, 2020; BRANDÃO SCS, et al., 2020). Um estudo de coorte retrospectivo realizado por Danying L, et al. (2020) em Wuhan, na China, evidenciou uma diminuição gradual de fibrinogênio ao longo do tempo de doença em pacientes não sobreviventes e um aumento substancial nos estágios iniciais da COVID-19, já que se trata de uma proteína reativa aguda sendo consumida durante a progressão da doença.

Além disso, nesse mesmo estudo foi observado uma diminuição na contagem de plaquetas em pacientes graves, refletindo seu consumo e geração de trombina, se demostrando útil no reconhecimento da presença e da gravidade da coagulopatia (DANYING L, et al., 2020). Outras anormalidades laboratoriais encontradas são o aumento da lactato desidrogenase (LDH), podendo alguns pacientes apresentar altas concentrações de ferritina. É importante ressaltar que a trombocitopenia não foi encontrada como um importante preditor da progressão da doença ou de desfecho adverso (LEVI M, et al., 2020).

Com base nas atuais evidências, é recomendado monitorar a coagulopatia em pacientes hospitalizados com infecção por COVID-19 recém-confirmada ou presuntiva através da contagem de plaquetas e concentrações de dímero-D a cada 2-3 dias (CONNORS JM e LEVY JH, 2020; LEVI M, et al., 2020). Ademais, é importante monitorar os fatores relacionados à internação, como a imobilização prolongada, que pode aumentar o risco de fenômenos tromboembólicos, além de verificar a necessidade de profilaxia com anticoagulantes de acordo com cada caso (LEVI M, et al., 2020).

Diante o exposto, as alterações laboratoriais mais características de uma evolução desfavorável e um pior prognóstico em paciente com COVID-19 associado a trombocitopenia foram: aumento da proporção de neutrófilos para linfócitos, tempo de protrombina prolongado e dímero-D aumentado (PRADO EM, et al., 2021; DANYING L, et al., 2020). Dessa maneira, sabe-se que a coagulopatia associada a COVID-19 está diretamente associada a um risco aumentado de morte (PRADO EM, et al., 2021). Sendo assim, é necessária a observação de parâmetros de coagulação para a identificação e intervenção da coagulopatia o mais precocemente possível, uma vez que, pacientes com COVID-19 grave e internados na UTI possuem um alto risco de trombose, já que apresentam uma prevalência de 45,6\% de tromboembolismo venoso (PRADO EM, et al., 2021; DANYING L, et al., 2020; LEENTJENS J, et al., 2021).

\section{Perfil dos pacientes}

Pacientes com COVID-19 que desenvolvem o tipo grave da doença normalmente apresentam anormalidades hemostáticas, as quais se assemelham a coagulopatia intravascular disseminada (CIVD) juntamente à sepse, mas com a diferença de que na presença do coronavírus há uma maior chance de trombose em vez de hemorragias (TIBURI RGB, et al., 2021). Além disso, as comorbidades são muito frequentes em pacientes graves. Dentre elas, a hipertensão arterial sistêmica (HAS) se apresenta com uma expressiva prevalência nos casos que agravaram em torno de $43,9 \%$ nos estados críticos. Ainda, a HAS é a comorbidade mais prevalente na população que vai a óbito quando comparada com os sobreviventes, sendo $52,6 \%$ a 18,2\% respectivamente (TIBURI RGB, et al., 2021; WANG J, et al., 2020).

O Diabetes Mellitus (DM) se demonstrou importante no agravamento da coagulopatia induzida pelo COVID-19 como consequência do estado pró-trombótico induzido pela hiperglicemia do paciente diabético. Da mesma forma, a idade avançada também se destaca como um importante fator de risco para complicações, sendo a média de idade dos pacientes graves entre 60-65 anos (TIBURI RGB, et al., 2021). Outro fator de risco para forma grave do coronavírus observado em muitos estudos é o sexo masculino, no qual a frequência varia de $84,2 \%$ em pacientes com COVID que foram a óbito e $46,6 \%$ naqueles que sobreviveram (WANG J, et al., 2020). Pacientes com comorbidades tendem a desenvolver complicações decorrentes da infecção pelo Coronavírus, principalmente os que apresentam: idade avançada, hipertensão arterial sistêmica (HAS) e diabetes mellitus (DM) (TIBURI RGB, et al., 2021). 
Dentre as coagulopatias comumente relacionadas a COVID-19, pode-se citar o tromboembolismo venoso, cujos fatores de risco estão relacionados a fisiopatologia da doença, como um estado inflamatório agudo, imobilização por um longo período de tempo e presença de outros fatores de risco cardiovascular (KOLLIAS A, et al., 2020). Verificou-se uma prevalência de aproximadamente $30 \%$ de tromboembolismo venoso (TEV), $20 \%$ de trombose venosa profunda (TVP) e de embolia pulmonar em 18\% dos pacientes com COVID-19 em sua forma grave (DI MINNO A, et al., 2020).

Um estudo realizado na Universidade de Nebraska demonstra que em grande quantidade dos pacientes testados positivos para o Sars-Cov-2 possuem contagem de Dímero-D elevada, sendo essa contagem ainda maior para pacientes que possuem alguma comorbidade, elevando o risco para desenvolver qualquer coagulopatia. Considerando os riscos, esse mesmo estudo considera importante a anticoagulação profilática para qualquer paciente com COVID-19 (DOBESH PP e TRUJILLO TC, 2020). É importante ressaltar que há relato de caso na literatura que indica a presença de tromboembolismo pulmonar em paciente de 22 anos e assintomático durante infecção pelo Sars-Cov-2.

\section{Manejo do paciente com coagulopatia na COVID-19}

Para todos os casos de pacientes com COVID-19 internados, o uso de heparina de baixo peso molecular (HBPM) em dose profilática é a primeira medida a ser adotada como profilaxia às suas principais coagulopatias. No entanto, ainda há controvérsias na literatura sobre os benefícios e recomendações em relação ao uso de dosagens intermediárias ou terapêuticas, quando adotadas para a tromboprofilaxia. Nos casos em que a profilaxia farmacológica esteja contraindicada, como em sangramentos ativos ou alto risco de sangramento, o uso de compressão pneumática intermitente e meias de compressão são recomendadas em pacientes graves (ZHAI Z, et al., 2020; THACHIL J, et al., 2020; GOSWAMI J, et al., 2021).

Em relação ao tratamento da TVP e embolia pulmonar (EP) na COVID-19, a primeira linha é a anticoagulação com HBPM em dose terapêutica. Contudo, em situações de comprometimento renal severo (clearance de creatinina $<30 \mathrm{ml} / \mathrm{min}$ ), é recomendado o uso de heparina não fracionada (HNF), com monitorização da atividade do anti-Xa e contagem de plaquetas, devido ao risco de trombocitopenia induzida por heparina (ZHAl Z, et al., 2020). As heparinas, além de seus efeitos anticoagulantes, também parecem estar associadas a efeitos anti-inflamatórios, diminuição da progressão do desenvolvimento de CID e inibição da replicação do Sars-CoV-2, por se ligar às suas proteínas spike (MIESBACH W e MAKRIS M, 2020; HADID T, et al., 2020; ZANZA C, et al., 2021).

Ademais, no caso de pacientes com sinais de EP maciça ou de alto risco, combinado a achados do ecocardiograma à beira do leito, é indicado a utilização de terapia trombolítica. Além disso, para pacientes criticamente graves, com colapso circulatório refratário ou parada cardíaca, pode-se ponderar o uso de oxigenação por membrana extracorpórea (ECMO) associado à embolectomia cirúrgica ou tratamento direcionado por cateter (ZHAl Z, et al., 2020).

$\mathrm{O}$ uso de anticoagulantes orais diretos (DOACs) e antagonistas da vitamina $\mathrm{K}$ possuem muitas limitações para TVP e, para EP, os DOACs se tornam opção apenas após sua fase aguda. Além disso, esses anticoagulantes orais possuem interação com determinados medicamentos antivirais, muitas vezes adotados em uso off label contra a COVID-19, o que aumenta o risco de sangramento (ZHAI Z, et al., 2020; MIESBACH W e MAKRIS M, 2020).

A utilização de antiagregantes plaquetários não é atualmente indicada para coagulopatias relacionadas a COVID-19 e sua associação às heparinas em dose terapêutica pode cursar com aumento de risco para sangramento (IBA T, et al., 2020c; GOSWAMI J, et al., 2021). Em relação ao uso off label de agentes fibrinolíticos, algumas séries de casos relataram melhora da oxigenação em pacientes com síndrome do desconforto respiratório agudo (SDRA) relacionados à COVID-19. Todavia, considerando-se os riscos da prática, seu uso segue não recomendado (ZANZA C, et al., 2021; GOSWAMI J, et al., 2021).

Embora a COVID-19 possa cursar com quadro semelhante à CID, sangramentos não parecem ser frequentes em decorrência da doença. No entanto, caso ocorram, o tratamento é o mesmo de pacientes não COVID-19, com transfusões e descontinuação ou reversão da anticoagulação, individualizando-se às 
necessidades do paciente. Devido ao aumento do risco trombótico, a reposição profilática de fatores de coagulação ou plaquetas não são recomendadas (ZANZA C, et al., 2021; HADID T, et al., 2020).

De um modo geral, além da terapia farmacológica, a monitorização dinâmica e atenta dos parâmetros sanguíneos e de coagulação, como a quantidade de neutrófilos e linfócitos, o número de plaquetas, dímero $\mathrm{D}$ e protrombina também é uma medida importante e eficaz para o manejo de um paciente acometido por coagulopatias. A constante classificação de risco é outro método confiável e conveniente para avaliar, gerenciar e predizer a gravidade dos indivíduos criticamente enfermos (DANYING L, et al., 2020). É válido ressaltar que mulheres grávidas têm risco 4 a 5 vezes maior de apresentar trombose em razão de alterações hormonais e mecânicas. Por esse motivo, o manejo desse tipo de paciente, quando acometida por COVID19, deve ser avaliado com cautela (LLAU JV, et al., 2020).

Após a alta hospitalar, pacientes com infecção por COVID-19 tratados com anticoagulantes devem manter a terapia farmacológica independentemente do curso da doença e de acordo com a indicação médica. Recomenda-se que o regime adotado seja baseado na administração medicamentosa por via parenteral. A HBPM é a escolha comumente aplicada na maioria dos casos por conta da facilidade da terapêutica e em razão da baixa necessidade de monitoramento, salvo em casos de insuficiência renal. $\mathrm{Na}$ ausência de informações qualificadas, a continuação de medidas farmacológicas deve ser conservada para os pacientes com maior risco (LLAU JV, et al., 2020; BIKDELI B, et al., 2020).

A recuperação e o acompanhamento do paciente acometido também se estendem a mecanismos de educação permanente para o automonitoramento dos sintomas. Uma explicação suficiente sobre o quadro clínico e cuidadosas considerações sobre o manejo da condição pode evitar visitas desnecessárias a hospitais. Uma alimentação rica em vitamina K, bem como uma dieta estável deve ser encorajada. Ademais, com a economia prejudicada em decorrência da pandemia, a desvantagem socioeconômica foi um fator recentemente associado a taxas mais elevadas de casos de coagulopatias. Essas perdas podem impactar negativamente a continuidade do tratamento após a alta hospitalar. Dessa forma, o debate sobre a questão bem como a proposição de políticas públicas para mitigá-la pode configurar uma ação importante para combater essa problemática (LLAU JV, et al., 2020; BIKDELI B, et al., 2020).

\section{Medidas preventivas}

Em pacientes de gravidade leve ou moderada é recomendado a hidratação adequada, movimentação e a profilaxia pós-alta (ZHAI Z, et al., 2020). Essa profilaxia consiste no acompanhamento do risco trombótico quando se fizer necessário. Dessa maneira, deve ser individualizada por meio da análise da idade do paciente, existência de condição de mobilidade reduzida, TEV anterior, IMC $>30$ e câncer ativo ou outras comorbidades pró-trombóticas. Na vigência de algum desses componentes, é recomendado a realização de exames laboratoriais para contagem de plaquetas, tempo de protrombina, fibrinogênio e dímero- $D$, além dos parâmetros inflamatórios IL-6, proteína C reativa, ferritina e procalcitonina (FRANCHINI M, et al., 2020). Segundo um estudo randomizado feito na Holanda, a terapia compressiva logo após o diagnóstico de TVP diminui em $20 \%$ a obstrução venosa, auxiliando na prevenção da síndrome pós-trombótica (AMIN EE, et al., 2018).

\section{CONSIDERAÇÕES FINAIS}

O novo coronavírus provoca distúrbios respiratórios além de diversas manifestações sistêmicas como as coagulopatias. Estas são decorrentes de uma resposta inflamatória exacerbada e vistas com maior frequência nos casos mais graves. Nesse sentido, torna-se necessário avaliar a presença de comorbidades nos pacientes acometidos pela COVID, visando identificar sobretudo a existência prévia de distúrbios de coagulação. Dessa maneira, com adequado rastreio e medidas profiláticas, é possível diminuir as chances de complicações graves e melhorar o cuidado aos pacientes. O estudo é limitado ao que pouco se sabe ainda sobre os mecanismos fisiopatológicos decorrentes das complicações de distúrbios coagulativos consequentes da infecção por COVID-19. Portanto, ainda há a demanda de maiores pesquisas quanto a doença no geral e suas variadas formas de distúrbios multissistêmicos que vem sendo relatada através de trabalhos mais atuais. 


\section{REFERÊNCIAS}

1. AMIN EE, et al. Reduced incidence of vein occlusion and postthrombotic syndrome after immediate compression for deep vein thrombosis. Blood, 2018; 132(21): 2298-2304.

2. BIKDELI B, et al. COVID-19 and Thrombotic or Thromboembolic Disease: Implications for Prevention, Antithrombotic Therapy, and Follow-Up: JACC State-of-the-Art Review. J Am Coll Cardiol, 2020; 75(23): 2950-2973.

3. BRANDÃO SCS, et al. COVID-19 grave: entenda o papel da imunidade, do endotélio e da coagulação na prática clínica. Jornal Vascular Brasileiro, 2020; 19: e20200131.

4. CARVALHO ACS, et al. Moduladores de coagulação alterados comprometem os pacientes infectados com COVID19. Brazilian Journal of Health Review, 2020; 3(5): 11624-11644

5. CONNORS JM, LEVY JH. COVID-19 and its implications for thrombosis and anticoagulation. Blood, 2020; 135(23): 2033-2040.

6. DANYING L, et al. Haematological characteristics and risk factors in the classification and prognosis evaluation of COVID-19: a retrospective cohort study. The Lancet Haematology, 2020; 7(9): e671-e678.

7. DI MINNO A, et al. COVID-19 and venous thromboembolism: a meta-analysis of literature studies. Seminars in Thrombosis Hemostasis, 2020; 46(7): 763-771.

8. DOBESH PP, TRUJILLO TC. Coagulopathy, venous thromboembolism, and anticoagulation in patients with COVID19. Pharmacotherapy, 2020; 40(11): 1130-1151.

9. FRANCHINI M, et al. COVID-19 associated coagulopathy. De Gruyter, 2020; 7(4): 357-363

10. GÓMEZ-MESA JE, et al. Thrombosis and Coagulopathy in COVID-19. Current problems in cardiology, 2021; 46(3): 100742.

11. GOSWAMI J, et al. A review of pathophysiology, clinical features, and management options of COVID-19 associated coagulopathy. Shock, 2021; 55(6): 700-716.

12. HADID T, et al. Coagulation and anticoagulation in COVID-19. Blood Reviews, 2021; 47: 100761.

13. HUANG C, et al. Clinical features of patients infected with 2019 novel coronavirus in Wuhan, China. The Lancet, 2020; 395 (10223): 497-506.

14. IBA T, et al. Coagulopathy in COVID-19. Journal of Thrombosis and Haemostasis, 2020b; 18(9): 2103-2109.

15. IBA T, et al. Coagulopathy of coronavirus disease 2019. Critical Care Medicine, 2020c; 48(9): 1358-1364.

16. IBA T, et al. The coagulopathy, endotheliopathy, and vasculitis of COVID-19. Inflammation Research, 2020a; 69(12): $1181-1189$.

17. KOLLIAS A, et al. Thromboembolic risk and anticoagulant therapy in COVID-19 patients: emerging evidence and call for action. British Journal of Haematology, 2020; 189(5): 846-847.

18. LEENTJENS J, et al. COVID-19-associated coagulopathy and antithrombotic agents-lessons after 1 year. The Lancet Haematology, 2021.

19. LEVI M, et al. Coagulation abnormalities and thrombosis in patients with COVID-19. The Lancet Haematology, 2020; 7(6): e438-e440.

20. LLAU JV, et al. Recomendaciones de consenso SEDAR-SEMICYUC sobre el manejo de las alteraciones de la hemostasia en los pacientes graves con infección por COVID-19, 2020; 67(7): 391-399.

21. MIESBACH W, MAKRIS M. COVID-19: Coagulopathy, Risk of Thrombosis, and the Rationale for Anticoagulation. Clinical and applied thrombosis/hemostasis, 2020; 26: 1-7.

22. NAQVI AAT, et al. Insights into SARS-CoV-2 genome, structure, evolution, pathogenesis and therapies: structural genomics approach. Biochimica et Biophysica Acta (BBA)-Molecular Basis of Disease, 2020; p. 165878.

23. PINTO TS, DE OLIVIEIRA JB. Distúrbios de coagulação em pacientes infectados por Sars-Cov-2. News Lab, 2021.

24. PRADO EM, et al. Repercussões hematológicas, cardiovasculares e pulmonares no prognóstico de pacientes infectados por COVID-19: uma revisão integrativa. Brazilian Journal of Health Review, 2021; 4(1): 1646-1668.

25. SINGH SP, et al. Microstructure, pathophysiology, and potential therapeutics of COVID-19: A comprehensive review. J Med Virol. 2021; 93(1): 275-299.

26. STEFELY JA, et al. Marked factor V activity elevation in severe COVID-19 is associated with venous thromboembolism. American Journal of Hematology, 2020; 95(12): 1522- 1530.

27. THACHIL J, et al. ISTH interim guidance on recognition and management of coagulopathy in COVID-19. Journal of Thrombosis and Haemostasis, 2020; 18(5): 1023-1026.

28. TIBURI RGB, et al. Coagulopatia induzida pelo estado inflamatório da infecção pela Covid-19. Brazilian Journal of Health Review, 2021; 4(2): 8478-8501.

29. WANG J, et al. Thrombo-inflammatory features predicting mortality in patients with COVID-19: the FAD-85 score. Journal of International Medical Research, 2020; 48(9): 1-14.

30. WORLD HEALTH ORGANIZATION (WHO). 2021a. In: WHO Coronavirus (COVID-19) Dashboard. Disponível em: https://covid19.who.int/ . Acesso em: 2 de julho de 2021.

31. WORLD HEALTH ORGANIZATION (WHO). 2021b. In: Brazil: WHO Coronavirus Disease. Disponível em: https://covid19.who.int/region/amro/country/br. Acesso em: 2 de julho de 2021.

32. WU C, et al. Risk Factors Associated With Acute Respiratory Distress Syndrome and Death in Patients With Coronavirus Disease 2019 Pneumonia in Wuhan, China. JAMA Intern Med. 2020; 180(7): 934-943.

33. ZANZA C, et al. Risk management and treatment of coagulation disorders related to COVID-19 infection. International Journal of Environmental Research and Public Health, 2021;18(3): 1268.

34. ZHAI Z, et al. Prevention and treatment of venous thromboembolism associated with coronavirus disease 2019 infection: a consensus statement before guidelines. Thrombosis and Haemostasis, 2020; 120(6): 937-948. 